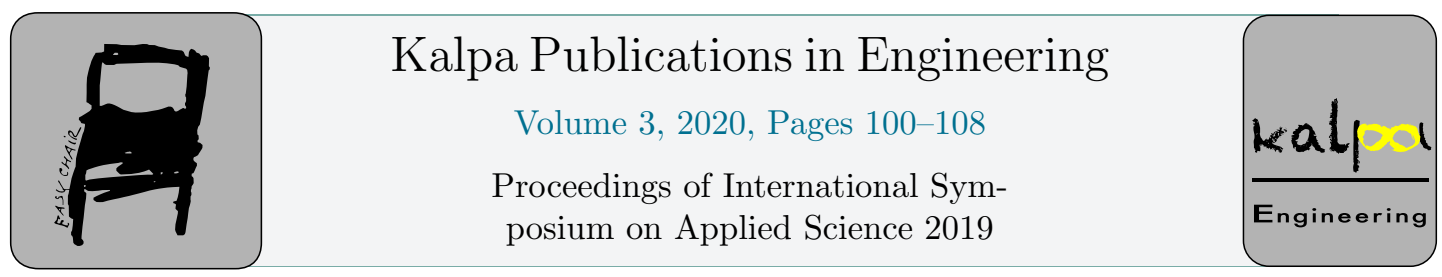

\title{
Exercise Physiology: Improving Stationary Bike Training Performance Using Heart Rate Variability
}

\author{
Quoc Cuong Pham ${ }^{1}$, Tran Duc Minh Nguyen ${ }^{1}$, Cao Dang Le ${ }^{1}$, Quoc Khai \\ Le $^{1}$, Quang Linh Huynh ${ }^{1}$ \\ ${ }^{1}$ Ho Chi Minh University of Technology - VNUHCM \\ 1510384@hcmut.edu.vn
}

\begin{abstract}
Exercising is said to bring benefits to people taking part in, not only physical but also physiological gain. Heart Rate Variability (HRV) is an important marker reflecting the function of autonomic nervous system (ANS), which has shown potentials in some exercise therapy and sport physiology studies. HRV analysis is said to be used for getting better understanding of our body's response to exercise and the reaction to different stressors from workout. Thus, it is essential to monitor and optimize the recovery to avoid overtraining. This study aims to investigate the influence of HRV reflecting the physical stress level on participants when exercising, therefore, building a concept of self-training guide to improve the adaptation and performance. Electrocardiogram (ECG) is acquired by BIOPAC system over 10 healthy college students during a proposed training protocol on stationary bike, and post-exercising. HRV data from ECG is analyzed in time, frequency and nonlinear domains to extract various features to evaluate physiological recovery status, manage physical fatigue, intensity adjustment. From the evaluation of these indexes, participants are able to keep track of their physiological condition as well as to have more effective training exercises.
\end{abstract}

\section{Introduction}

Exercise physiology is the study of body's acute responses and chronic adaptations to exercise. Those responses and adaptations include changes in muscular, cardiovascular, and neurohumoral systems that lead to changes in functional capacity and strength due to endurance training or strength training. The effect of training on the body has been defined as the reaction to the adaptive responses of the body arising from exercise or as "an elevation of metabolism produced by exercise" [1]. Over the past few decades several studies have shown that participation in regular physical activity is associated with a decrease in all-cause mortality, particularly from cardiovascular disease (CVD) [2]. 
Conversely, there are ample reports indicating that vigorous physical exertion is associated with an increased risk for cardiac events, including sudden cardiac death (SCD) in individuals harboring CVD [3]. Physical activity (PA) and exercise have been extensively recognized as important influences on the relationship between psychosocial stress and CVD probably because of its influence on physical fitness. Thus, it would be expected that more active individuals, who conversely possess higher physical fitness, would be more resilient to mental stresses [4]. Chandola et al. (2008) reported a positive association between work stress and CVD possibly as a consequence of the negative influence of stress on health behaviors (i.e., reduced PA and poor diet) [5]. As a result, exercising needs to be supervised, where electrocardiography is a powerful tool.

Electrocardiography (ECG) is a technique in which electrode is placed over the skin to record electrical activity of the heart. For millennia we have understood that heart rate (HR) responds to stress. Two recent developments allow one to examine measures of this linkage more precisely: the first development differentiates between chronic stress exposure and responses to short-term stressors, while the second development deals with different ways of characterizing the fundamental properties of the HR itself and different ways of measuring its variability [6]. Ongoing troubles and the failure to resolve negative emotional states such as anger and anxiety generate imbalance between the sympathetic (SNS) and the parasympathetic nervous system (PNS), the two branches of the autonomic nervous system (ANS). An increase in the sympathetic-to-parasympathetic ratio (SPR) is now being linked to increased cardiovascular morbidity and mortality. Heart rate variability (HRV) is an easy and non-invasive tool for the assessment of variations in beat-to-beat intervals and autonomic nervous system activity with HRV obtained by linear methods within the domains of time and frequency analyses, and nonlinear methods. HRV, which is claimed to reflect the activity of ANS, has been widely used in studies to: examine the effect of dehydration after exercise heat stress [7]; predict both cardiovascular and allcause mortality independent of clinical factors and exercise responses [8]; compare the influence of chronic and short-term stress [6]. Wilhelm von Rosenberg et al. (2017) resolved the ambiguities in the $\mathrm{LF} / \mathrm{HF}$ ratio, as it has received some criticism as a measure of cognitive and physical aspects of stress, and gave in-depth understanding of HRV by using LF - HF Scatter plot to categorize different stress state [9].

The purpose of the study is to: i) examine the relationship between both physical and mental stress and training performance using HRV; ii) give suggestions on how to improve training performance.

\section{Material and Methods}

The empty space on top of the first page, just before the title, will be reserved for a page header ECG acquired through MP36 (BIOPAC) with a 3-electrode set and a BSL software run on a laptop with a sampling rate of $200 \mathrm{~Hz}$. A chest strap is used to record ECG with II electrode Lead attached on their chests throughout the procedure. 10 male subjects have taken part in the training procedure, who are around 20 having different physical activity (PA) levels and no concerning medical issue.

The entire procedure takes after of what has been done in the previous study [10], which is described below: Before starting a 2-month cycling exercise, participants are surveyed by a questionnaire to have an overview of their health consisting of physical activity habit, medical record and aspiration. A program is designed based on 4 training factors: Type, Intensity, Frequency and Duration [11]. The stationary bike workout lasts for 2 months with resistance is adjusted according to students' performance to keep their heart working at 55-65\% maximum heart rate zone (maximum heart rate is calculated by equation in [11]). Students are asked to train at least 3 days per week and bike 30 minutes per training day at minimum with constant speed at 60 round-per-minute (RPM) [11] [12]. As they finish their cycling, they are asked to stop and rest on bike for 3 more minutes to measure the recovery 
rate. Their pre-training and post-training conditions are examined as extra information for analysis. After every 3 training days, $\mathrm{VO}_{2}$ max is measured by Step Test, which is designed by Queens College.

exercising is that it would create noise due to motion, sweating chest strap, etc., which poses difficulty in finding R peaks. In order to get the best HRV data, the proposed method is as follow: the 30-min ECG data is divided equally into 2 blocks, and for each block is then divided into 5-second windows to check Hjorth activity value. Each block is statistically calculated relative threshold based on the popular Hjorth activity value range in all windows. Hence, each window would be classified as stable, which needs no correction, or unstable, which would be skipped as the HRV value at this window is extremely high. Last step is to interpolate all the abnormal beat in HRV (Fig. 1).
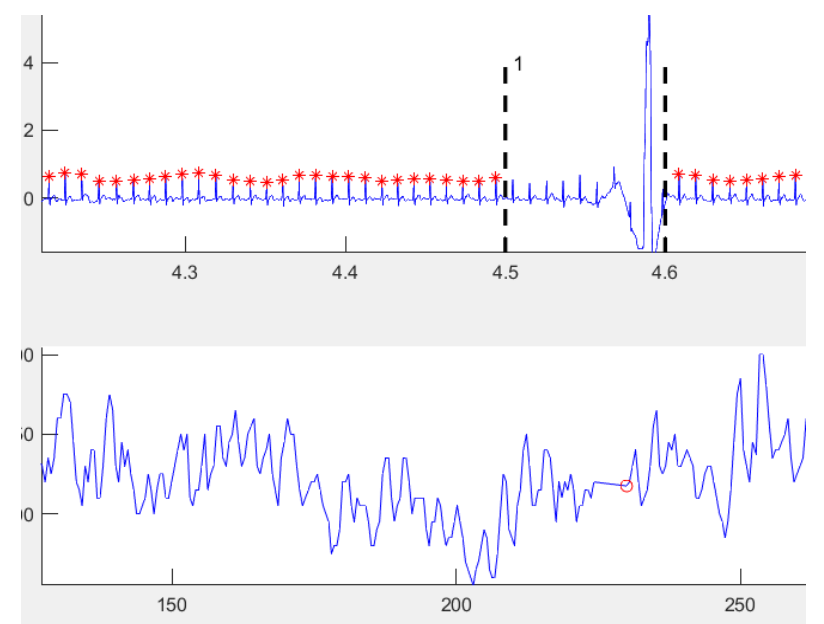

Figure 1: Noise generated in HRV measured while exercising

After preprocessing, HRV analysis open source code of Marcus Vollmer are used to calculate some basic HRV parameters in time (SDNN, RMSSD), frequency (LF, HF and LF/HF ratio) and nonlinear domain (SD1, SD2) [13]. These indices are then plot and compare between training days of each participants. Additionally, the indices used in study [10] are also considered to evaluate the relationship of physical stress and training performance. Hence, recommendations are proposed on how to improve exercising performance and monitoring.

\section{Results and Discussion}

Frequency domain

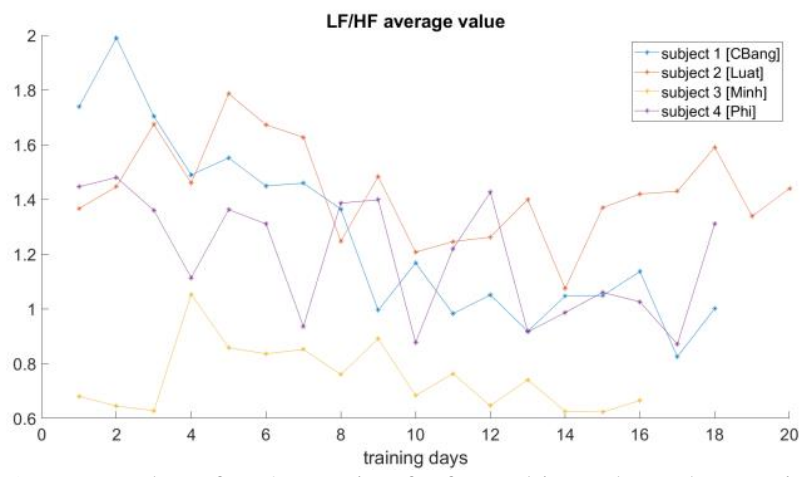

Figure 2: Average value of $\mathrm{LF} / \mathrm{HF}$ ratio of a few subjects throughout training procedure 
The general trend of LF/HF ratio is the more people exercise or expose to physical activity, the closer to range $0.8-1$ the ratio. Study has shown that the body at rest, or balance, when the ratio is about 0.8 and would be higher if body is exposed to stress. The power of LF clearly higher than that of HF indicates the body is going through physical stress such as exercising. However, if the power of HF is higher than LF, it shows that the body is having mental stress including sorrow event, worries or any uncomfortable feelings. Additionally, HRV-related studies also show that the power of lower oscillations highly correlated to the activity of SNS and that of higher frequency correlated to PNS activity. As a result, keeping a ratio balance means keeping the balance between PNS and SNS, which can say that the training brought positive outcomes.

However, as can be seen in Fig. 2, there are some cases show no improvements such as subject 2. In this case, subject 2 for the first day of training, the LF/HF ratio is 1.37 and the last day is 1.44 , which is opposite the desired trend. As mentioned in the previous part where the heart rate of the participant is kept between $55-65 \%$ maximum heart rate zone (Fig. 3), the level of weight was adjusted to satisfy that condition. So, in this case, the improvement of the participant is not shown by the LF/HF ratio but by the weight level. The subject could endure the same physical stress for higher intensity.

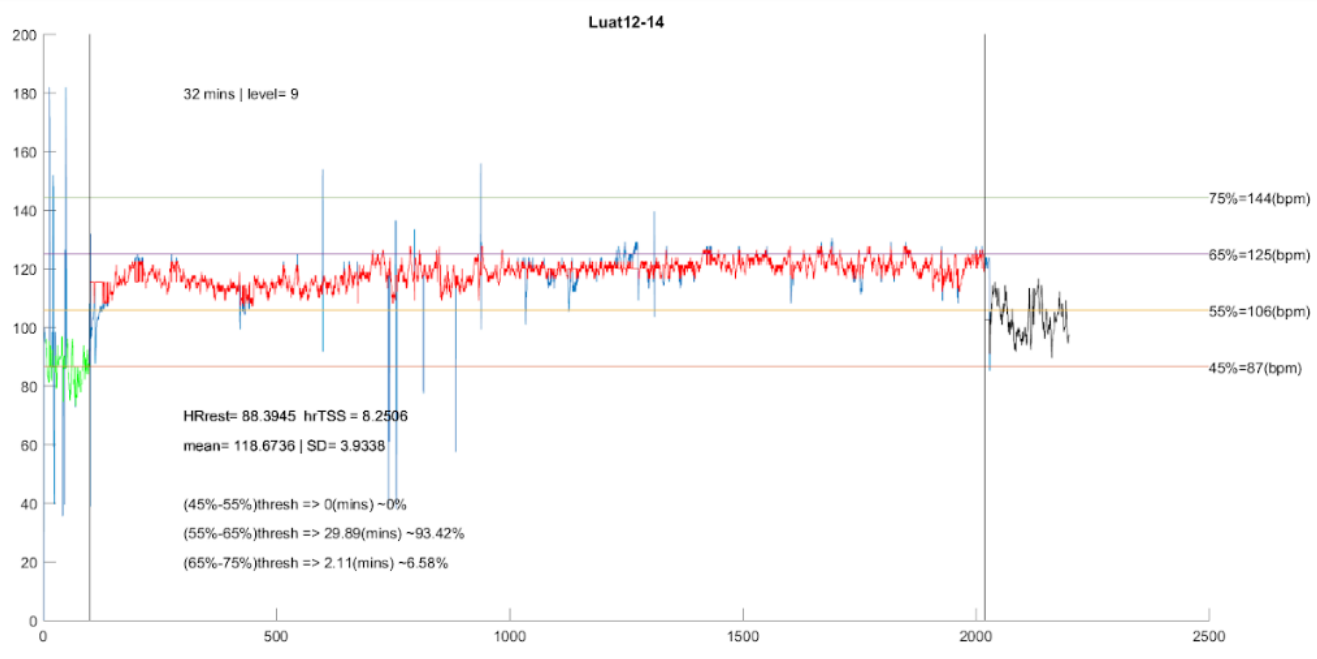

Figure 3: Heart rate of subject 2 on the first day (above) and last day of training (below)

The same circumstance can be seen in subject 3 in Fig. 2, it shows no positive outcome and the ratio levelized at approximately 0.65 at the end of the training, which indicates the subject has been going through mental stress almost the entire procedure. According to the survey which was made by the subject every day before training, it was reported that the subject 3 had insufficiency of sleep, cold and negative feelings. Figure 4 shows the LF - HF Scatter plot, which was used in [9] to differentiate the stress states, of the subject in the last day of training. The power of HF ranges from 5 to 7.5 (ms2) and the power of LF ranges from 3.5 to 4.5 (ms2) stating that the participant suffered from mental stress, which could be derived from reasons mentioned above. In this case, the performance of the subject was compromised with the stress he exhibited to. 
Table 1. Changes in LF/HF ratio in all subjects

\begin{tabular}{c|ccc}
\hline Subject & $\begin{array}{l}\text { LF/HF } \\
\text { before }\end{array}$ & $\begin{array}{l}\text { LF/HF } \\
\text { after }\end{array}$ & Percentage \\
\hline 1 & 1.74 & 1.00 & $-42.53 \%$ \\
2 & 1.37 & 1.07 & $-21.90 \%$ \\
3 & 0.68 & 0.62 & $-8.82 \%$ \\
4 & 1.45 & 1.03 & $-28.97 \%$ \\
5 & 0.74 & 0.78 & $5.41 \%$ \\
6 & 0.83 & 0.96 & $15.66 \%$ \\
7 & 1.06 & 0.75 & $-29.25 \%$ \\
8 & 1.04 & 1.19 & $14.42 \%$ \\
9 & 1.26 & 1.23 & $-2.38 \%$ \\
10 & 1.75 & 1.49 & $-14.86 \%$ \\
\hline
\end{tabular}

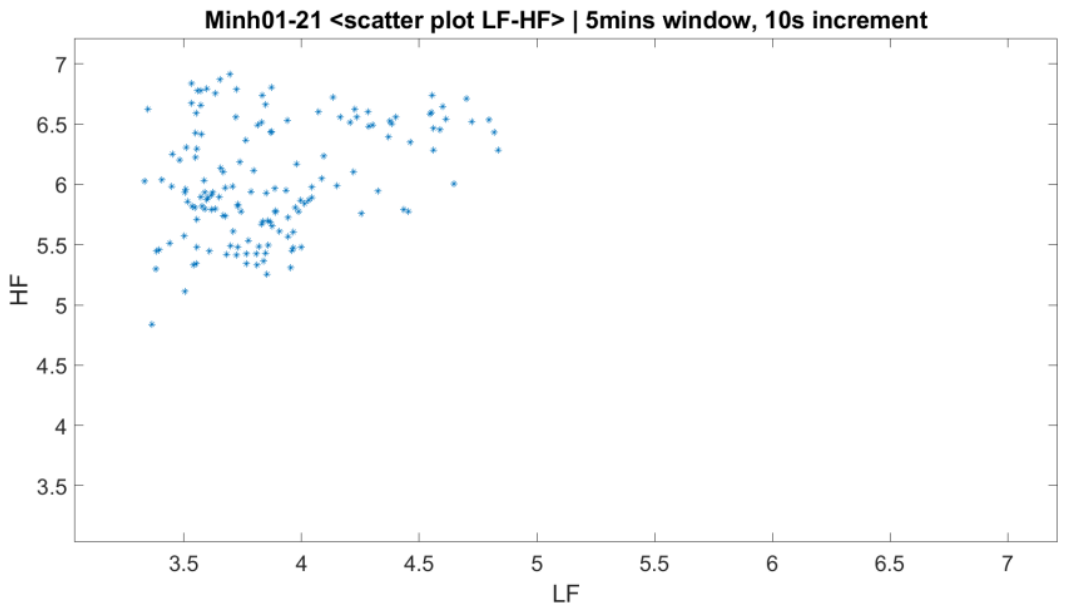

Figure 4: LF - HF Scatter plot of subject 3 in one training day. Y-axis is the power of HF and X-axis is the

Time domain power of LF.

The HRV of most subjects increases at the end of the training procedure compared to the beginning. This is a good sign because higher HRV means lower heart rate. It also means that the heart becomes stronger and adapt better to the same level of training intensity, which was proven to be true in the study [10]. In the study mentioned, the cardiac endurance improvement was measured through the VO2max and HR recovery value increase depending on the performance of each subject. In Fig. 5, though HRV is expected to rise at the end of the training procedure, there are still some cases pose different pattern. Subject 5 has a drop of $12.80 \%$ of HRV (Table 2), which probably rooted from physical stress such as being exposed to higher exercise intensity. As HRV reflects more of psychological aspect of stress than of physiological, the main cause contributing to the downward trend is the mental side. As mentioned above, subject 5 who suffered from mental stress during 2 training months has negative outcome for the $\mathrm{LF} / \mathrm{HF}$ ratio, it also affects to the $\mathrm{HRV}$ value of the subject. 


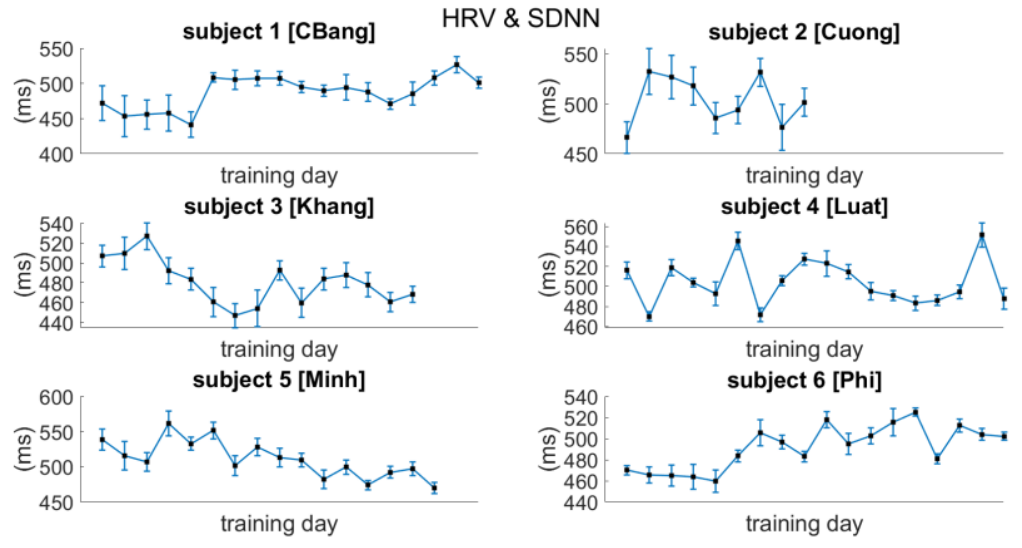

Figure 5: HRV value (black dot) and SDNN value (blue vertical line) of 6 subjects

Table 2. Changes in HRV in all subjects

\begin{tabular}{c|ccc}
\hline Subject & HRV before & HRV after & Percentage \\
\hline 1 & 471.89 & 500.88 & $6.14 \%$ \\
2 & 466.20 & 501.62 & $7.60 \%$ \\
3 & 506.98 & 468.30 & $-7.63 \%$ \\
4 & 470.20 & 551.47 & $17.28 \%$ \\
5 & 538.59 & 469.63 & $-12.80 \%$ \\
6 & 465.45 & 512.36 & $10.08 \%$ \\
7 & 442.52 & 467.42 & $5.63 \%$ \\
8 & 441.84 & 488.80 & $10.63 \%$ \\
9 & 471.01 & 476.34 & $1.13 \%$ \\
10 & 496.12 & 474.88 & $-4.28 \%$ \\
\hline
\end{tabular}
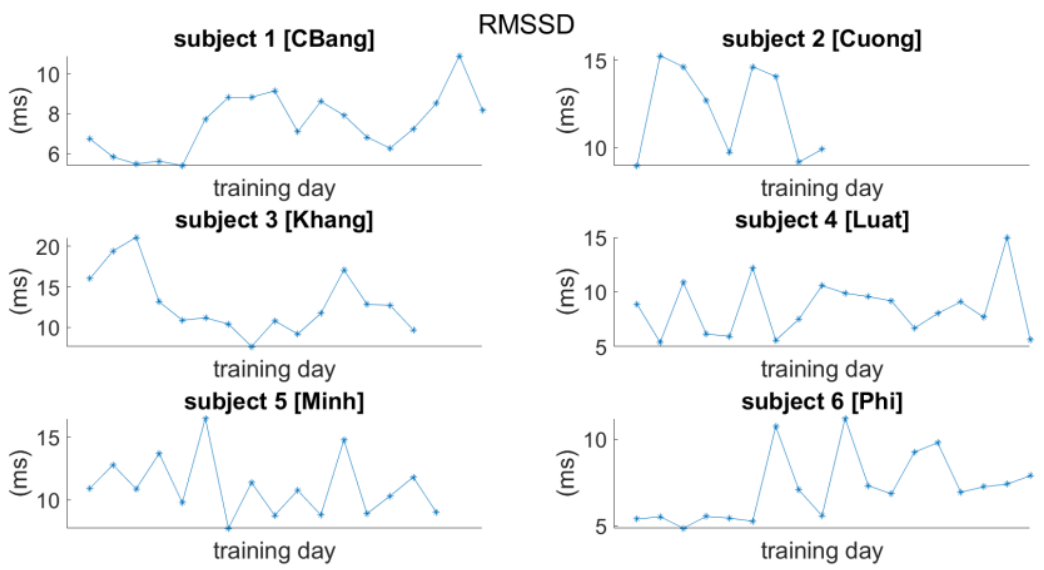

Figure 6: RMSSD value of 6 subjects throughout the training

SDNN and RMSSD indices are also calculated among subjects and and they elicit a downward trend in general (Fig. 5 and Fig. 6). These two indices are usually used to predict cardiac risk and sudden 
death in epilepsy, the lower they are, the higher risk of CVD the subject has to be specific. Also these indices are usually measured subject at rest and in $24 \mathrm{~h}$ recording, unlike in this study. In sum, further in-depth study needed to specify the meaning of these measurements recorded while exercising.

Nonlinear domain

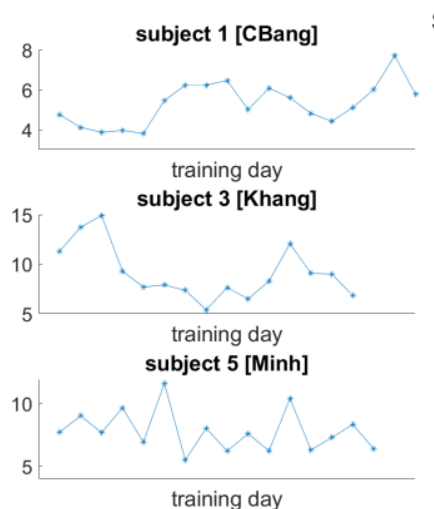

SD1
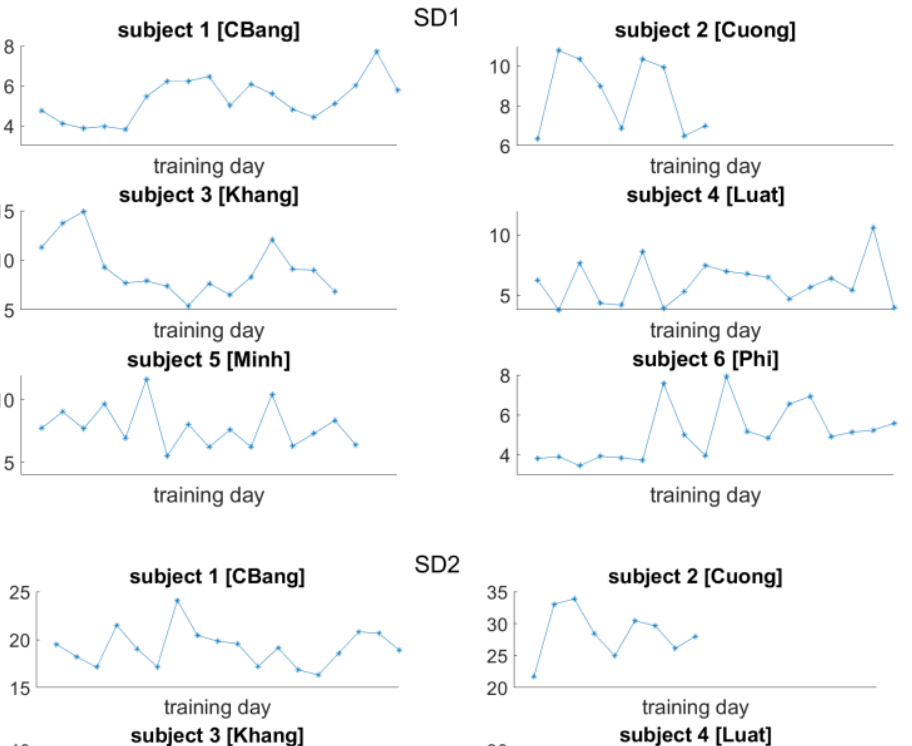

SD2
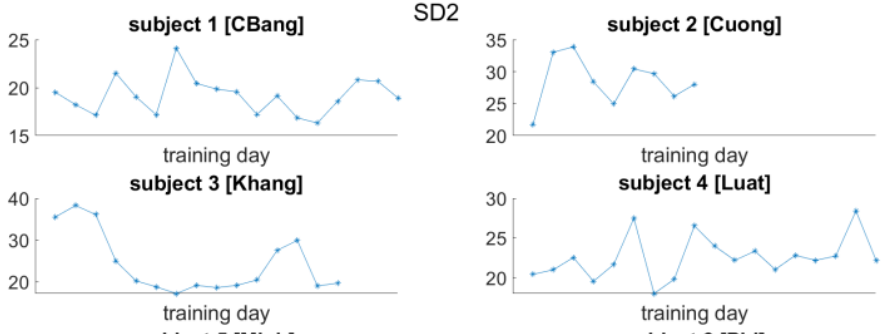

subject 5 [Minh]
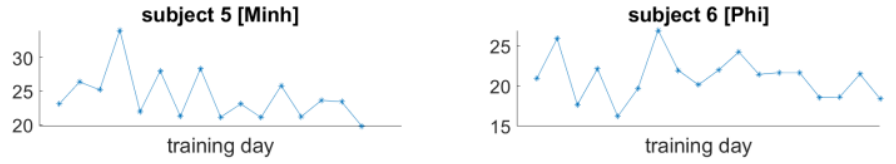

Figure 7. SD1 (above) and SD2 (below) value of 6 subjects

Both SD1 and SD2 value of all subjects vary differently and show no trend. It can be inferred that training has no effect on the nonlinear parameters of HRV.

\section{Conclusions}

In sum, the study aims to use HRV to evaluate the influence of stress, both physical and mental, on the performance of the trainees. By analyzing the HRV in time, frequency and nonlinear domain, it is clear that though exercising on stationary bike helps students to gain better physical and physiological attributes [10], it could be sanitated if we take into the stress. From the result above, we could see that exercising help to balance the autonomous nervous system and able to withstand more workload. Furthermore, HRV also reflects the mental stress that the students going through and reduce the efficiency of training. As can be seen there are a few subjects were under stress most probably from study or work who cannot gain as most as other subjects. It is recommended that to get the most of 
benefit from training, subjects should, firstly, be monitored regularly to keep the heart working in the desired heart rate zone. Either it is tranining for endurance or strength, the heart needs to be pushed hard enough to have improvements by changing resistance, speed or time. HRV is expected to drop when intially increase the workload, but it would rise again when the heart get used to the change. The LF/HF ratio might be high at first but it would gradually decline as the heart adapt better to higher workload. The second recommendation is that trainees needs to be surveyed and examined about physical and mental stress. Both aspects would affect to the performance of each day and in general. For instance, a few subjects above were suffered from mental stress which negatively affect to the performance of that day. Hence, when evaluate the improvement of exercising, it is better to consider both aspects, physical parameters such as VO2max, blood pressure, heart rate recovery, etc. and psychological parameters like LF/HF ratio, HRV value, SDNN, and so on. Some students show improvement in one side not in the other, and vice versa.

The drawback of this study is that it is not able to find any relationship or correlation of the nonlinear parameters to the performance. And it does not take into account the impact of environment where training takes place and the nutrition of the subjects.

\section{References}

Awtry, Eric H.; Balady, Gary J., "Exercise and Physical Activity," in Textbook of Cardiovascular Medicine (3rd ed.), 2007, p. 83.

Christine M. Albert, Murray A. Mittleman, Claudia U. Chae, I.-Min Lee, Charles H. Hennekens, Joann E. Manson, "Triggering of Sudden Death from Cardiac Causes by Vigorous Exertion," The New England Journal of Medicine, vol. 343, p. 1355-1361, 2000.

Jesse A. Berlin, Graham A. Colditz, "A meta-analysis of physical activity in the prevention of coronary heart disease," American Journal of Epidemiology, vol. 132, no. 4, p. 612-628, 1990.

M. Hamer, "Psychosocial Stress and Cardiovascular Disease Risk: The Role of Physical Activity," American Psychosomatic Society, pp. 896-903, 2012.

Tarani Chandola, Annie Britton, Eric Brunner, Harry Hemingway, Marek Malik, Meena Kumari, Ellena Badrick, Mika Kivimaki, and Michael Marmot, "Work stress and coronary heart disease: what are the mechanisms?," European Heart Journal, pp. 640-648, 2008.

C. Schubert, M. Lambertz, R.A. Nelesen, W. Bardwell, J.-B. Choi, J.E. Dimsdale, "Effects of stress on heart rate complexity-A comparison between short-term and chronic stress," Biological Psychology, no. 80, pp. 325-332, 2009.

Robert Carter III, Samuel N. Cheuvront, D.Walter Wray, Margaret A. Kolka, Lou A. Stephenson, Michael N. Sawka, "The influence of hydration status on heart rate variability after exercise heat stress," Journal of Thermal Biology, no. 30, pp. 495-502, 2005.

Frederick E. Dewey, BA, James V. Freeman, MD, Gregory Engel, MD, Raul Oviedo, MD, Nayana Abrol, MD, Natasha Ahmed, MD, Jonathan Myers, PhD, and Victor F. Froelicher, MD, "Novel predictor of prognosis from exercise stress testing: Heart rate variability response to the exercise treadmill test," American Heart Journal, pp. 281-288, 2007.

Wilhelm von Rosenberg, Theerasak Chanwimalueang, Tricia Adjei, Usman Jaffer, Valentin Goverdovsky and Danilo P. Mandic, "Resolving Ambiguities in the LF/HF Ratio: LF-HF Scatter Plots for the Categorization of Mental and Physical Stress from HRV," Frontiers in Physiology, vol. VIII, 2017.

P.Q. Cuong, N.T.D. Minh, L.C. Dang, "Exercise physiology application: Cardiac endurance training for students by stationary bike," in SEATUC Symposium, Ha Noi, 2019. 
William J. Kraemer, Steven J. Fleck, Michael R. Deschenes, "Aerobic and Strength Training Prescription for Health and Performance," in Exercise Physiology Intergrating Theory and Application, Philadelphia, Wolters Kluwer Health, 2012, pp. 362-380.

T. Battinelli, "Aerobic and Anaerobic Conditioning," in Physique, Fitness, and Performance, Boca Raton, CRC Press, 2007, pp. 75-79.

M. Vollmer, "MathWorks," 1 September 2019. [Online]. Available: https://www.mathworks.com [Accessed 20 August 2019]. 